\title{
Positive solutions for semilinear elliptic equations with critical weighted Hardy-Sobolev exponents *
}

\author{
Hai-Yan Liu Chun-Lei Tang ${ }^{\dagger}$
}

\begin{abstract}
In this paper, we study a class of semilinear elliptic equations with critical weighted Hardy-Sobolev exponents and superlinear nonlinearity. By means of the variational methods and some analysis techniques, positive solution is obtained.
\end{abstract}

\section{Introduction and main results}

Consider the following semilinear elliptic problem

$$
\begin{cases}-\operatorname{div}\left(|x|^{-2 a} \nabla u\right)-\mu \frac{u}{|x|^{2(1+a)}}=\frac{|u|^{p-2}}{|x|^{b p}} u+f(x, u), & x \in \Omega \\ u=0, & x \in \partial \Omega\end{cases}
$$

where $\Omega$ is an open bounded domain in $\mathbb{R}^{N}(N \geq 3)$ with smooth boundary $\partial \Omega$ and $0 \in \Omega, 0 \leq a<\sqrt{\bar{\mu}}, 0 \leq \mu<(\sqrt{\bar{\mu}}-a)^{2}$ with $\bar{\mu} \triangleq \frac{(N-2)^{2}}{4}, a \leq b<a+1$, $p=p(a, b) \triangleq \frac{2 N}{N-2(1+a-b)}$ is the Hardy-Sobolev critical exponent. And note that

*Supported by National Natural Science Foundation of China(No. 11471267)

†Corresponding author.

Received by the editors in July 2014.

Communicated by D. Bonheure.

2010 Mathematics Subject Classification : 35J25; 35J20; 35J61; 35J75.

Key words and phrases : weighted Hardy-Sobolev exponents, Mountain Pass Lemma, semilinear elliptic equation. 
$p(a, a)=\frac{2 N}{N-2}=2^{*}$ is the Sobolev critical exponent. $f \in C(\bar{\Omega} \times \mathbb{R}, \mathbb{R}), F(x, t)$ is a primitive function of $f$ defined by $F(x, t)=\int_{0}^{t} f(x, s) d s$ for $x \in \Omega, t \in \mathbb{R}$.

In the case $\mu=0$, problem (1) is related to the well known Caffarelli-KohnNirenberg inequalities in [1],

$$
\left(\int_{\mathbb{R}^{N}}|x|^{-b p}|u|^{p} d x\right)^{\frac{2}{p}} \leq C_{a, b} \int_{\mathbb{R}^{N}}|x|^{-2 a}|\nabla u|^{2} d x, \text { for all } u \in C_{0}^{\infty}\left(\mathbb{R}^{N}\right)
$$

where $0 \leq a<\sqrt{\bar{\mu}}, a \leq b<a+1, p=\frac{2 N}{N-2(1+a-b)}$. As well known, CaffarelliKohn-Nirenberg inequalities contain the classical Sobolev inequality $(a=b=0)$ and the Hardy inequality $(a=0, b=1)$ as special cases and have played a crucial role in many applications by virtue of the complete knowledge about the $C_{a, b}$, thus it is a fundamental task to study problem (1). As $b=1+a$ and $p=2$ in (2), there is the following weighted Hardy- inequality [2],

$$
\int_{\mathbb{R}^{N}} \frac{|u|^{2}}{|x|^{2(1+a)}} d x \leq \frac{1}{(\sqrt{\bar{\mu}}-a)^{2}} \int_{\mathbb{R}^{N}} \frac{|\nabla u|^{2}}{|x|^{2 a}} d x, \text { for all } u \in C_{0}^{\infty}\left(\mathbb{R}^{N}\right) .
$$

Moreover, as $a=0$, (3) becomes the well known Hardy inequality,

$$
\int_{\mathbb{R}^{N}} \frac{|u|^{2}}{|x|^{2}} d x \leq \frac{1}{\bar{\mu}} \int_{\mathbb{R}^{N}}|\nabla u|^{2} d x, \text { for all } u \in C_{0}^{\infty}\left(\mathbb{R}^{N}\right) .
$$

For $\mu \in\left[0,(\sqrt{\bar{\mu}}-a)^{2}\right)$, we use $H=H_{0}^{1}\left(\Omega,|x|^{-2 a}\right)$ to denote the completion of $C_{0}^{\infty}(\Omega)$ with respect to the norm,

$$
\|u\| \triangleq\left(\int_{\Omega}\left(|x|^{-2 a}|\nabla u|^{2}-\mu \frac{u^{2}}{|x|^{2(1+a)}}\right) d x\right)^{\frac{1}{2}},
$$

which is equivalent to the usual norm of $H_{0}^{1}\left(\Omega,|x|^{-2 a}\right)$ due to (3). And also we can define the best Hardy-Sobolev constant:

$$
A=A_{a, b, u}(\Omega) \triangleq \inf _{u \in H \backslash\{0\}} \frac{\|u\|^{2}}{\left(\int_{\Omega} \frac{|u|^{p}}{|x|^{b p}} d x\right)^{\frac{2}{p}}} .
$$

In recent years, people have paid much attention to the existence of solutions for this singular problems concerning the operator $-\Delta \cdot-\frac{\mu}{|x|^{2}} \cdot \quad(0 \leq \mu<\bar{\mu})$ with Sobolev critical exponents (the case that $a=b=0$ see $[3,12-16]$ and there references). Some authors also studied the singular problems with HardySobolev critical exponents(the case that $a=0, b \neq 0$ see $[5,11,17-21]$ ). In $[19,22,23]$ the authors only studied the special cases of $f(x, t)$, for example, in $[19], f(x, t)=\lambda|t|^{q-2} t$ with suitable $q$ and in $[22,23], f(x, t)=\lambda t$. Besides, there are some authors who studied the general form. In [17] Ding and Tang discussed the case of $a=0$ and $b \neq 0$. After that, in [4] Huang, Wu and Tang discussed the problem with $a \neq 0$ and $b \neq 0$ concave-convex nonlinearities. It should be 
mentioned that in the case $a=b=\mu=0$, Brezis and Nirenberg discussed the problem (see [9])

$$
\begin{cases}-\Delta u=u^{2^{*}-1}+f(x, u), & \text { on } \Omega \\ u>0, & \text { on } \Omega \\ u=0, & \text { on } \partial \Omega\end{cases}
$$

and obtained one positive solution for suitable $f$.

In the case $a=0, b \neq 0, \mu \neq 0$, Kang and Peng discussed the problem (see [19])

$$
\begin{cases}-\Delta u-\mu \frac{u}{|x|^{2}}=\frac{|u|^{2^{*}(s)-2}}{|x|^{s}} u+\lambda|u|^{q-2} u, & x \in \Omega \\ u=0, & x \in \partial \Omega\end{cases}
$$

for $0 \leq \mu<\bar{\mu}, 0 \leq s<2, \lambda>0,2 \leq q<2^{*}$ and obtained the following results.

Theorem 1.(Theorem 1.1 in [19]) Suppose that $0 \leq s<2,0 \leq \mu<\bar{\mu}, \lambda>0$, and

$$
\max \left\{2, \frac{N}{\sqrt{\bar{\mu}}+\sqrt{\bar{\mu}-\mu}}, \frac{N-2 \sqrt{\bar{\mu}-\mu}}{\sqrt{\bar{\mu}}}\right\}<q<2^{*} .
$$

Then problem (5) has a one positive solution in $H_{0}^{1}(\Omega)$.

After that, in [17], Ding and Tang deal with the following problem with general form

$$
\begin{cases}-\Delta u-\mu \frac{u}{|x|^{2}}=\frac{|u|^{2^{*}(s)-2}}{|x|^{s}} u+f(x, u), & x \in \Omega \backslash\{0\} \\ u=0, & x \in \partial \Omega\end{cases}
$$

for $0 \leq \mu<\bar{\mu}, 0 \leq s<2$ and obtained one positive solution as following

Theorem 2.(Theorem 1 in [17]) Suppose that $N \geq 3,0 \leq \mu<\sqrt{\bar{\mu}}, 0 \leq s<2$,

$\left(f_{1}\right) f \in C\left(\bar{\Omega} \times \mathbb{R}^{+}, \mathbb{R}\right), \lim _{t \rightarrow 0^{+}} \frac{f(x, t)}{t}=0$, and $\lim _{t \rightarrow+\infty} \frac{f(x, t)}{t^{2^{*}-1}}=0$, uniformly for $x \in \bar{\Omega}$;

$\left(f_{2}\right)$ There exists a constant $\rho, \rho>2$, such that $0<\rho F(x, t) \leq f(x, t)$ t for all $x \in \bar{\Omega}$, $t \in \mathbb{R}^{+} \backslash\{0\}$.

Assume that

$$
\rho>\max \left\{2, \frac{N}{\sqrt{\bar{\mu}}+\sqrt{\bar{\mu}-\mu}}, \frac{N-2 \sqrt{\bar{\mu}-\mu}}{\sqrt{\bar{\mu}}}\right\} .
$$

Then problem (6) has at least one positive solution.

By the same methods, the case $\mu \neq 0, a \neq 0, b \neq 0$, Huang, Wu and Tang discussed the problem (see [6])

$$
\begin{cases}-\operatorname{div}\left(|x|^{-2 a} \nabla u\right)-\mu \frac{u}{|x|^{2(1+a)}}=\frac{|u|^{p-2}}{|x|^{b p}} u+f(x, u), & x \in \Omega \backslash\{0\} \\ u=0, & x \in \partial \Omega\end{cases}
$$

for $0 \leq a<\sqrt{\bar{\mu}}, 0 \leq \mu<(\sqrt{\bar{\mu}}-a)^{2}, a \leq b<a+1$ and obtained one positive solution as following 
Theorem 3.(Theorem 1 in [6]) Suppose that $N \geq 3(1+a), 0 \leq a<\sqrt{\bar{\mu}}, 0 \leq \mu<$ $(\sqrt{\bar{\mu}}-a)^{2}, a \leq b<a+1$, and there exists a constant $\rho>2$ such that

$\left(f_{3}\right) f \in C\left(\bar{\Omega} \times \mathbb{R}^{+}, \mathbb{R}\right), \lim _{t \rightarrow 0^{+}} \frac{f(x, t)}{t^{\rho-1}}=\sigma>0$, and $\lim _{t \rightarrow+\infty} \frac{f(x, t)}{t^{2^{*}-1}}=0$, uniformly for $x \in \bar{\Omega}$;

$\left(f_{4}\right) 0<\rho F(x, t) \leq f(x, t) t$ for all $x \in \bar{\Omega}, t \in \mathbb{R}^{+} \backslash\{0\}$. Assume that

$$
\rho>\max \left\{2, \frac{N}{\gamma}, \frac{N-2 \beta}{\sqrt{\bar{\mu}}-a}\right\},
$$

where $\beta \triangleq \sqrt{(\sqrt{\bar{\mu}}-a)^{2}-\mu}$ and $\gamma \triangleq \sqrt{\bar{\mu}}-a+\beta$. Then problem (7) has at least one positive solution.

Note that the Ambrosetti-Rabinowitz condition is crucial importance to the existence of weak solutions in [6] and [17]. In fact, [9] has obtained the existence of solutions without Ambrosetti-Rabinowitz condition. It is natural to ask whether problem (1) admits one solutions without the Ambrosetti-Rabinowitz condition. In this present paper, we use a variational method to deal with problem (1) with general form and superlinearities, we give a positive answer.

The main difficulty in dealing with problem (1) is that the corresponding functional does not satisfy the (PS) condition due to the lack of compactness of the embedding in $H \hookrightarrow L^{2^{*}}(\Omega)$ and the computation of $s u p_{t \geq 0} I(t u)$. Hence we could not use standard variational methods. However, a local (PS) condition can be established in a suitable range. Then the existence result is obtained via constructing a minimax level within this range, and the Mountain Pass Lemma due to Rabinowitz [7].

Throughout this paper, we will denote the norms on $H$ and $L^{q}(\Omega)$ respectively by $\|\cdot\|,\|\cdot\|_{L^{q}}$. Different positive constants will be denoted by the $M, M_{i}$ or $C_{i}(i=1,2, \cdots)$ for convenience.

Here are the main results of this paper:

Theorem 4. Suppose that $0 \leq a<\sqrt{\bar{\mu}}, p=\frac{2 N}{N-2(1+a-b)}, 0 \leq \mu<(\sqrt{\bar{\mu}}-a)^{2}$, $a \leq b<a+1,0<\lambda<\lambda_{1}, \lambda_{1}$ is the first eigenvalue of - div $\left(|x|^{-2 a} \nabla \cdot\right)-\mu|x|^{-2(1+a)}$, and $f$ satisfies the conditions

$\left(f_{5}\right) f \in C\left(\bar{\Omega} \times \mathbb{R}^{+}, \mathbb{R}\right), \limsup _{t \rightarrow 0^{+}} \frac{F(x, t)}{t^{2}}<\frac{1}{2} \lambda$, and $\lim _{t \rightarrow+\infty} \frac{F(x, t)}{t^{p}}=0$, uniformly for $x \in \bar{\Omega}$.

Then problem (1) has at least one positive solution for every $f$ under one of the following conditions:

(a) For $N>2 a+2+2 \sqrt{\mu+(1+a)^{2}}$, there exist positive constant $\sigma$, a nonempty open subset $\omega$ with $0 \in \omega \subset \Omega$ and a nonempty open interval $I \subset(0,+\infty)$, so that $f(x, t) \geq 0$ for almost everywhere $x \in \omega$ and for all $t \geq 0, f(x, t) \geq \sigma>0$ for almost everywhere $x \in \omega$ and for all $t \in I$;

(b) For $N=2 a+2+2 \sqrt{\mu+(1+a)^{2}}$, there exist positive constants $\sigma, D$ and $a$ nonempty open subset $\omega$ with $0 \in \omega \subset \Omega$, so that $f(x, t) \geq 0$ for almost everywhere $x \in \omega$ and for all $t \geq 0 ; f(x, t) \geq \sigma$ for almost everywhere $x \in \omega$ and for all $t \in[0, D]$ 
or $f(x, t) \geq \sigma t$ for almost everywhere $x \in \omega$ and for all $t \in[D,+\infty]$;

(c) For $3(1+a) \leq N<2 a+2+2 \sqrt{\mu+(1+a)^{2}}$, there exists a nonempty open subset $\omega$ with $0 \in \omega \subset \Omega$, so that $f(x, t) \geq 0$ for almost everywhere $x \in \omega$ and for all $t \geq 0$ and $\lim _{t \rightarrow+\infty} \frac{f(x, t)}{t^{\frac{N-\gamma^{\prime}-3 \beta}{\gamma^{\prime}+\beta}}}=+\infty$ uniformly as $x \in \omega$.

Remark 1. In this paper, we obtain the similar results as those in [6], while the conditions of $f$ are different from [6], and the conditions of $f$ are better than [6] when $b=a, p=2^{*}$, it also extended [17] and [19] when $s=0$. Besides, we see the results of this paper, find it contains [9] when $b=a=0, p=2^{*}$ and $\mu=0$. There are some functions $f$ satisfying the assumptions of our paper but not satisfying those in [6]. For example, let

$$
f(x, t)=\left\{\begin{array}{r}
\sigma+\omega_{1}(x)|t|^{r_{1}-2} t, \text { for all } N>2 a+2+2 \sqrt{\mu+(1+a)^{2}}, \\
\sigma t+\omega_{1}(x)|t|^{r_{1}-2} t, \text { for all } N=2 a+2+2 \sqrt{\mu+(1+a)^{2}} \\
\omega_{1}(x)|t|^{r_{1}-2} t+\omega_{2}(x)|t|^{r_{2}-2} t \\
\text { for all } 3(1+a) \leq N<2 a+2+2 \sqrt{\mu+(1+a)^{2}},
\end{array}\right.
$$

where $\omega_{1}, \omega_{2}>0$ and $\omega_{1}, \omega_{2} \in C(\bar{\Omega}), \sigma>0$, and $2<r_{1}<p, 3<r_{2}<p$. Moreover, we obtain the existence of positive solutions for problem (1) by the Mountain Pass Theorem of Ambrosetti and Rabinowitz without the (PS) condition.

\section{Proof of theorem}

It is obvious that the values of $f(x, t)$ for $t<0$ are irrelevant in Theorem 4 , and we may define

$$
f(x, t)=0, \quad \text { for } x \in \Omega, \quad t \leq 0 .
$$

In order to study the existence of positive solutions for problem (1), we shall firstly consider the existence of nontrivial solutions to the problem

$$
\begin{cases}-\operatorname{div}\left(|x|^{-2 a} \nabla u\right)-\mu \frac{u}{|x|^{2(1+a)}}=\frac{\left(u^{+}\right)^{p-1}}{|x|^{b p}}+f\left(x, u^{+}\right), & x \in \Omega \backslash\{0\}, \\ u=0, & x \in \partial \Omega,\end{cases}
$$

where $u^{+}=\max \{u, 0\}$. The energy functional corresponding to problem (8) is given by

$$
\begin{aligned}
I(u)=\frac{1}{2} \int_{\Omega}\left(|x|^{-2 a}|\nabla u|^{2}-\mu \frac{u^{2}}{|x|^{2(1+a)}}\right) d x- & \frac{1}{p} \int_{\Omega} \frac{\left(u^{+}\right)^{p}}{|x|^{b p}} d x- \\
& \int_{\Omega} F\left(x, u^{+}\right) d x, \quad \text { for any } u \in H .
\end{aligned}
$$

By the weighted Hardy-Sobolev inequality and $\left(f_{5}\right), I \in C^{1}(H, \mathbb{R})$. Now it is well known that there exists a one to one correspondence between the weak solutions 
of problem (8) and the critical points of $I$ on $H$. More precisely we say that $u \in H$ is a weak solution of problem (8), if for any $v \in H$, there holds

$$
\begin{aligned}
& \left\langle I^{\prime}(u), v\right\rangle=\int_{\Omega}\left(|x|^{-2 a}(\nabla u, \nabla v)-\mu \frac{u v}{|x|^{2(1+a)}}\right) d x- \\
& \qquad \int_{\Omega} \frac{\left(u^{+}\right)^{p-1} v}{|x|^{b p}} d x-\int_{\Omega} f\left(x, u^{+}\right) v d x=0 .
\end{aligned}
$$

Let $\left\{u_{n}\right\}$ be a sequence in $H$ and $c \in \mathbb{R},\left\{u_{n}\right\}$ is called to be a (PS) $)_{c}$ sequence in $H$ if $I\left(u_{n}\right) \rightarrow c, I^{\prime}\left(u_{n}\right) \rightarrow 0$ in $H^{*}$ as $n \rightarrow \infty$, where $H^{*}$ is the dual space of $H$. We say that $I$ satisfies the (PS) $)_{c}$ condition if any $(\mathrm{PS})_{c}$ sequence $\left\{u_{n}\right\} \subset H$ has a convergent subsequence.

Recently, it has been proved that problem (1) has positive solutions in [10] for suitable parameters $a, b$ and $\mu$. Moreover, they proved that $A$ is attained when $\Omega=\mathbb{R}^{N}$ by the functions

$$
y_{\varepsilon}(x)=\frac{\left(2 \varepsilon p \beta^{2}\right)^{\frac{1}{p-2}}}{|x|^{\prime}\left(\varepsilon+|x|^{(p-2) \beta}\right)^{\frac{2}{p-2}}}
$$

for all $\varepsilon>0,0 \leq a<\sqrt{\bar{\mu}}, 0 \leq \mu<(\sqrt{\bar{\mu}}-a)^{2}, a \leq b<a+1$, and where $\gamma^{\prime} \triangleq \sqrt{\bar{\mu}}-a-\beta, \beta \triangleq \sqrt{(\sqrt{\bar{\mu}}-a)^{2}-\mu}$. Moreover, the function $y_{\varepsilon}(x)$ solves the equation

$$
-\operatorname{div}\left(|x|^{-2 a} \nabla u\right)-\mu \frac{u}{|x|^{2(1+a)}}=\frac{|u|^{p-2}}{|x|^{b p}} u, \text { in } \mathbb{R}^{N} \backslash\{0\}
$$

Let

$$
C_{\varepsilon}=\left(2 \varepsilon p \beta^{2}\right)^{\frac{1}{p-2}}, \quad U_{\varepsilon}=\frac{y_{\varepsilon}}{C_{\varepsilon}}
$$

Define a cut-off function $\psi \in C_{0}^{\infty}(\omega)$ such that $\psi(x)=1$ for $|x| \leq R, \psi(x)=0$ for $|x| \geq 2 R, 0<\psi(x)<1$, where $B_{2 R}(0) \subset \Omega$. Set $u_{\varepsilon}(x)=\psi(x) U_{\varepsilon}(x), v_{\varepsilon}(x)=$ $\frac{u_{\varepsilon}(x)}{\left(\int_{\Omega}\left|u_{\varepsilon}(x)\right|^{p}|x|^{-b p} d x\right)^{\frac{1}{p}}}$, so that $\int_{\Omega}\left|v_{\mathcal{E}}(x)\right|^{p}|x|^{-b p} d x=1$. Then the following results can be obtained by the methods used in [11]:

$$
A+C_{1} \varepsilon^{\frac{2}{p-2}} \leq\left\|v_{\mathcal{E}}\right\|^{2} \leq A+C_{2} \varepsilon^{\frac{2}{p-2}}
$$

and

$$
\left\{\begin{array}{l}
C_{3} \varepsilon^{\frac{\xi}{p-2}} \leq \int_{\Omega}\left|v_{\varepsilon}(x)\right|^{\xi} d x \leq C_{4} \varepsilon^{\frac{\xi}{p-2}}, \quad 1 \leq \xi<\frac{N}{\gamma}, \\
C_{3} \varepsilon^{\frac{\xi}{p-2}}|\ln \varepsilon| \leq \int_{\Omega}\left|v_{\varepsilon}(x)\right|^{\xi} d x \leq C_{4} \varepsilon^{\frac{\xi}{p-2}}|\ln \varepsilon|, \quad \xi=\frac{N}{\gamma}, \\
C_{3} \varepsilon^{\frac{N-\xi(\sqrt{\bar{\mu}}-a)}{(p-2) \beta}} \leq \int_{\Omega}\left|v_{\mathcal{E}}(x)\right|^{\xi} d x \leq C_{4} \varepsilon^{\frac{N-\xi(\sqrt{\bar{\mu}}-a)}{(p-2) \beta}}, \quad \frac{N}{\gamma}<\xi<2^{*},
\end{array}\right.
$$


where $\gamma \triangleq \sqrt{\bar{\mu}}-a+\beta$. Moreover, since $H \hookrightarrow L^{p}(\Omega)\left(2<p \leq 2^{*}\right)$, by (9), we have

$$
\begin{aligned}
\int_{\Omega}\left|v_{\mathcal{E}}(x)\right|^{p} d x & \leq C\left(\int_{\Omega}\left|\nabla v_{\mathcal{E}}(x)\right|^{2} d x\right)^{\frac{p}{2}} \\
& \leq C\left(A+C_{2} \varepsilon^{\frac{2}{p-2}}\right)^{\frac{N}{N-2(1+a-b)}} \\
& \leq C_{5}(2 A)^{\frac{N}{N-2(1+a-b)}}\left(\varepsilon \rightarrow 0^{+}\right)
\end{aligned}
$$

so we obtain

$$
\int_{\Omega}\left|v_{\mathcal{\varepsilon}}(x)\right|^{p} d x \leq C_{5}(2 A)^{\frac{N}{N-2(1+a-b)}}, \quad \text { as } \varepsilon \rightarrow 0^{+} .
$$

Furthermore, we can get

$$
\begin{aligned}
& \int_{\Omega}\left|u_{\varepsilon}(x)\right|^{p}|x|^{-b p} d x \\
& =\int_{\Omega} \frac{\psi^{p}(x)}{|x|^{p\left(\gamma^{\prime}+b\right)}\left(\varepsilon+|x|^{(p-2) \beta)^{\frac{2 p}{p-2}}}\right.} d x \\
& =\int_{\Omega} \frac{1}{|x|^{p\left(\gamma^{\prime}+b\right)}\left(\varepsilon+|x|^{(p-2) \beta)^{\frac{2 p}{p-2}}}\right.} d x+\int_{\Omega \backslash B_{R}(0)} \frac{\psi^{p}(x)-1}{|x|^{p\left(\gamma^{\prime}+b\right)}\left(\varepsilon+|x|^{(p-2) \beta}\right)^{\frac{2 p}{p-2}}} d x \\
& =\int_{\mathbb{R}^{N}} \frac{1}{|x|^{p\left(\gamma^{\prime}+b\right)}\left(\varepsilon+|x|^{(p-2) \beta)^{\frac{2 p}{p-2}}}\right.} d x- \\
& \int_{\mathbb{R}^{N} \backslash \Omega} \frac{1}{|x|^{p\left(\gamma^{\prime}+b\right)}\left(\varepsilon+|x|^{(p-2) \beta)^{\frac{2 p}{p-2}}}\right.} d x+O(1) \\
& \leq \int_{\mathbb{R}^{N}} \frac{1}{|x|^{p\left(\gamma^{\prime}+b\right)}\left(\varepsilon+|x|^{(p-2) \beta)^{\frac{2 p}{p-2}}}\right.} d x-\int_{\mathbb{R}^{N} \backslash \Omega} \frac{1}{2^{\frac{2 p}{p-2}}|x|^{p\left(\gamma^{\prime}+b+2 \beta\right)}} d x+O(1) \\
& =\int_{\mathbb{R}^{N}} \frac{1}{|x|^{p\left(\gamma^{\prime}+b\right)}\left(\varepsilon+|x|^{(p-2) \beta)^{\frac{2 p}{p-2}}}\right.} d x-\int_{c}^{\infty} \frac{r^{N-1}}{2^{\frac{2 p}{p-2}} r^{p\left(\gamma^{\prime}+b+2 \beta\right)}} d r+O(1) \\
& =\int_{\mathbb{R}^{N}} \frac{1}{|x|^{p\left(\gamma^{\prime}+b\right)}\left(\varepsilon+|x|^{(p-2) \beta)^{\frac{2 p}{p-2}}}\right.} d x-\int_{c}^{\infty} \frac{2^{-\frac{2 p}{p-2}}}{r^{p \beta+1}} d r+O(1) \\
& \leq \int_{\mathbb{R}^{N}} \frac{\varepsilon^{\frac{N}{(p-2) \beta}}}{\varepsilon^{\frac{p\left(\gamma^{\prime}+b\right)}{(p-2) \beta}-\frac{2 p}{p-2}}|S|^{p\left(\gamma^{\prime}+b\right)}\left(1+|s|^{(p-2) \beta)^{\frac{2 p}{p-2}}}\right.} d s+O(1) \\
& =\varepsilon^{-\frac{p\left(\gamma^{\prime}+b+2 \beta\right)-N}{(p-2) \beta}} \int_{\mathbb{R}^{N}} \frac{1}{|s|^{p\left(\gamma^{\prime}+b\right)}\left(1+|s|^{(p-2) \beta)^{\frac{2 p}{p-2}}}\right.} d s+O(1) \\
& =\varepsilon^{-\frac{p}{p-2}} \int_{0}^{\infty} \frac{r^{N-1}}{r^{p\left(\gamma^{\prime}+b\right)}(1+r(p-2) \beta)^{\frac{2 p}{p-2}}} d r+O(1) \\
& \leq \varepsilon^{-\frac{p}{p-2}}\left(\int_{0}^{1} \frac{1}{r^{1-p \beta}} d r+\int_{1}^{\infty} \frac{1}{r^{p \beta+1}} d r\right)+O(1) \\
& \leq C_{6} \varepsilon^{-\frac{p}{p-2}} \text {, }
\end{aligned}
$$


where $c$ is some positive constant. Using the same method, we can also obtain

$$
\begin{aligned}
& \int_{\Omega}\left|u_{\varepsilon}(x)\right|^{p}|x|^{-b p} d x \\
& =\int_{\mathbb{R}^{N}} \frac{1}{|x|^{p\left(\gamma^{\prime}+b\right)}\left(\varepsilon+|x|^{(p-2) \beta)^{\frac{2 p}{p-2}}}\right.} d x- \\
& \int_{\mathbb{R}^{N} \backslash \Omega} \frac{1}{|x|^{p\left(\gamma^{\prime}+b\right)}\left(\varepsilon+|x|^{(p-2) \beta)^{\frac{2 p}{p-2}}}\right.} d x+O(1) \\
& \geq \int_{\mathbb{R}^{N}} \frac{1}{|x|^{p\left(\gamma^{\prime}+b\right)}\left(\varepsilon+|x|^{(p-2) \beta)^{\frac{2 p}{p-2}}}\right.} d x-\int_{\mathbb{R}^{N} \backslash \Omega} \frac{1}{|x|^{p\left(\gamma^{\prime}+b+2 \beta\right)}} d x+O(1)
\end{aligned}
$$

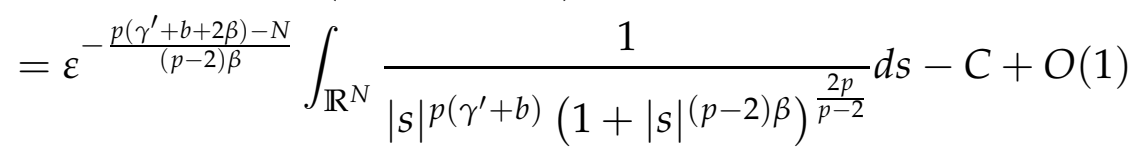

$$
\begin{aligned}
& =\varepsilon^{-\frac{p}{p-2}} \int_{0}^{\infty} \frac{r^{N-1}}{r^{p\left(\gamma^{\prime}+b\right)}\left(1+r^{(p-2) \beta}\right)^{\frac{2 p}{p-2}}} d r-C+O(1) \\
& \geq \varepsilon^{-\frac{p}{p-2}}\left(\int_{0}^{1} \frac{2^{-\frac{2 p}{p-2}}}{r^{1-p \beta}} d r+\int_{1}^{\infty} \frac{2^{-\frac{2 p}{p-2}}}{r^{p \beta+1}} d r\right)-C+O(1) \\
& \geq C_{7} \varepsilon^{-\frac{p}{p-2}} \text {. }
\end{aligned}
$$

So, one has

$$
C_{7 \varepsilon^{-\frac{p}{p-2}}} \leq \int_{\Omega}\left|u_{\varepsilon}(x)\right|^{p}|x|^{-b p} d x \leq C_{6} \varepsilon^{-\frac{p}{p-2}} .
$$

Lemma 1. Suppose $0 \leq a<\sqrt{\bar{\mu}}, a \leq b<a+1,0<\lambda<\lambda_{1}$. Assume $\left(f_{5}\right)$ and one of (a), (b) or (c) hold. Then there exists $u^{\prime} \in H, u^{\prime} \neq \equiv 0$, such that

$$
\sup _{t \geq 0} I\left(t u^{\prime}\right)<\frac{p-2}{2 p} A^{\frac{p}{p-2}}
$$

Proof. Consider the functions

$$
\begin{aligned}
& k(t)=I\left(t v_{\mathcal{\varepsilon}}\right)=\frac{t^{2}}{2}\left\|v_{\mathcal{\varepsilon}}\right\|^{2}-\frac{t^{p}}{p}-\int_{\Omega} F\left(x, t v_{\varepsilon}\right) d x, \\
& \widetilde{k}(t)=\frac{t^{2}}{2}\left\|v_{\mathcal{\varepsilon}}\right\|^{2}-\frac{t^{p}}{p} .
\end{aligned}
$$

Note that $\lim _{t \rightarrow+\infty} k(t)=-\infty, k(0)=0, k(t)>0$, as $t \rightarrow 0^{+}$, so $\sup _{t \geq 0} k(t)$ is attained for some $t_{\varepsilon}>0$.

First, we prove that $t_{\varepsilon}$ is bounded. When $x \in \Omega \backslash \omega$, we know

$$
U_{\mathcal{\varepsilon}}(x)=\frac{1}{|x|^{\gamma^{\prime}}\left(\varepsilon+|x|^{(p-2) \beta)^{\frac{2}{p-2}}}\right.} \leq \frac{1}{|x|^{\prime}\left(2|x|^{(p-2) \beta)^{\frac{2}{p-2}}}\right.}
$$


so $U_{\mathcal{\varepsilon}}(x)$ is bounded when $x \in \Omega \backslash \omega$, and by the definition of $v_{\mathcal{E}}(x)$, it is bounded, too. Without loss of generality, assume that there exists a positive constant $M_{0}<+\infty\left(M_{0}\right.$ is independent of $\left.\varepsilon\right)$, such that $\left|v_{\varepsilon}(x)\right| \leq M_{0}$. Moreover, from the condition of $\left(f_{5}\right)$, there exist constants $\varepsilon_{1}=\frac{1}{2} \lambda_{1} M_{0}^{2}, \varepsilon_{2}<\frac{1}{2 p}$, such that

$$
\left|F\left(x, t v_{\varepsilon}\right)\right| \leq \frac{1}{2} \lambda_{1}\left(t v_{\mathcal{\varepsilon}}\right)^{2}+\frac{\varepsilon_{2}}{M_{0}^{p}}\left(t v_{\mathcal{E}}\right)^{p} \leq \varepsilon_{1} t^{2}+\varepsilon_{2} t^{p}, x \in \Omega \backslash \omega
$$

and then we can directly get

$$
\begin{aligned}
k(t) & =\frac{t^{2}}{2}\left\|v_{\mathcal{\varepsilon}}\right\|^{2}-\frac{t^{p}}{p}-\int_{\Omega} F\left(x, t v_{\mathcal{\varepsilon}}\right) d x \\
& \leq \frac{t^{2}}{2}\left\|v_{\mathcal{\varepsilon}}\right\|^{2}-\frac{t^{p}}{p}-\int_{\Omega \backslash \omega} F\left(x, t v_{\mathcal{\varepsilon}}\right) d x \\
& \leq\left(\frac{\left\|v_{\varepsilon}\right\|^{2}}{2}+\varepsilon_{1}\right) t^{2}-\left(\frac{1}{p}-\varepsilon_{2}\right) t^{p} \\
& \leq t^{2}\left(\left(A+\varepsilon_{1}\right)-\frac{t^{p-2}}{2 p}\right) .
\end{aligned}
$$

Let $M_{1}=\left(2 p\left(A+\varepsilon_{1}\right)\right)^{\frac{1}{p-2}}+1$, then we get $k(t)<0$ for $t \geq M_{1}$, so by the definition of $k(t)$, we have

$$
t_{\varepsilon} \leq M_{1}
$$

It means that $t_{\varepsilon}$ has an upper bound. Now, we prove that $t_{\varepsilon}$ also has a lower bound. Let $\varepsilon_{3}=\left(2 C_{5}(2 A)^{\frac{N}{N-2(1+a-b)}}\right)^{-1}$. By $\left(f_{5}\right)$ and $0<\lambda<\lambda_{1}$, it is easy to verify that

$$
|f(x, t)| \leq \varepsilon_{3}|t|^{p-1}+\lambda_{1}|t| \text {. }
$$

Hence,

$$
0=k^{\prime}\left(t_{\varepsilon}\right)=t_{\varepsilon}\left(\left\|v_{\varepsilon}\right\|^{2}-t_{\varepsilon}^{p-2}-\frac{1}{t_{\varepsilon}} \int_{\Omega} f\left(x, t_{\varepsilon} v_{\varepsilon}\right) v_{\varepsilon} d x\right)
$$

we obtain

$$
\begin{aligned}
\left\|v_{\varepsilon}\right\|^{2} & =t_{\varepsilon}{ }^{p-2}+\frac{1}{t_{\varepsilon}} \int_{\Omega} f\left(x, t_{\varepsilon} v_{\varepsilon}\right) v_{\varepsilon} d x \\
& \leq t_{\varepsilon}^{p-2}+\varepsilon_{3} \int_{\Omega}\left|t_{\varepsilon}\right|^{p-2}\left|v_{\varepsilon}\right|^{p} d x+\lambda_{1} \int_{\Omega}\left|v_{\varepsilon}\right|^{2} d x .
\end{aligned}
$$

So by (11), one also has

$$
\begin{aligned}
& t_{\varepsilon}^{p-2}+\varepsilon_{3} \int_{\Omega}\left|t_{\varepsilon}\right|^{p-2}\left|v_{\varepsilon}\right|^{p} d x \\
= & t_{\varepsilon}^{p-2}\left(1+\varepsilon_{3} \int_{\Omega}\left|v_{\varepsilon}\right|^{p} d x\right) \\
\leq & t_{\varepsilon}^{p-2}\left(1+\varepsilon_{3} C_{5}(2 A)^{\frac{N}{N-2(1+a-b)}}\right) \\
\leq & \frac{3}{2} t_{\varepsilon}^{p-2} .
\end{aligned}
$$


Besides, from (10), we get

$$
\int_{\Omega}\left|v_{\mathcal{E}}\right|^{2} d x \rightarrow 0, \quad \text { as } \varepsilon \rightarrow 0
$$

Hence, we obtain

$$
\int_{\Omega}\left|v_{\mathcal{E}}\right|^{2} d x \leq \frac{A}{4 \lambda_{1}}
$$

which together with (13) and the left formula of (9), we get

$$
A \leq\left\|v_{\varepsilon}\right\|^{2} \leq \frac{3}{2} t_{\varepsilon}^{p-2}+\frac{1}{4} A,
$$

that is

$$
t_{\varepsilon}^{p-2} \geq \frac{A}{2}
$$

when $\varepsilon$ is sufficiently small.

Second, we compute $\sup _{t \geq 0} \widetilde{k}(t)$. Now we claim

$$
\left\|v_{\mathcal{E}}\right\|^{\frac{2 p}{p-2}} \leq A^{\frac{p}{p-2}}+C_{7} \varepsilon^{\frac{2}{p-2}}
$$

In order to prove this, we first prove the following inequality

$$
(a+b)^{r} \leq a^{r}+r(a+1)^{r-1} b, a>0, \quad r \geq 1
$$

In fact, set

$$
\varphi(x)=(a+x)^{r}-a^{r}-r(a+1)^{r-1} x, \quad 0 \leq x \leq 1 .
$$

Clearly, $\varphi^{\prime}(x) \leq 0$ for all $x \in(0,1)$, so $\varphi(b) \leq \varphi(0)=0$, then (16) holds. Let $a=A, b=C_{2} \varepsilon^{\frac{2}{p-2}}, r=\frac{p}{p-2}$, from (9) we have

$$
\left\|v_{\mathcal{\varepsilon}}\right\|^{\frac{2 p}{p-2}} \leq\left(A+C_{2} \varepsilon^{\frac{2}{p-2}}\right)^{\frac{p}{p-2}} \leq A^{\frac{p}{p-2}}+C_{8} \mathcal{E}^{\frac{2}{p-2}} .
$$

Thus our claim is true. From the definition of $\widetilde{k}(t)$, we know

$$
\widetilde{k}^{\prime}(t)=t\left(\left\|v_{\mathcal{\varepsilon}}\right\|^{2}-t^{p-2}\right)
$$

Setting $t_{\varepsilon}^{\prime}=\left\|v_{\varepsilon}\right\|^{\frac{2}{p-2}}$, we can get $\widetilde{k}^{\prime}\left(t_{\varepsilon}^{\prime}\right)=0$ and $\widetilde{k}^{\prime}(t) \geq 0$ for all $t \in\left[0, t_{\varepsilon}^{\prime}\right]$. So we conclude that $\sup _{t \geq 0} \widetilde{k}(t)$ attains at $t_{\varepsilon}^{\prime}$ and $\widetilde{k}$ is increasing in the interval $\left[0, t_{\varepsilon}^{\prime}\right]$, and it is easy to obtain

$$
\widetilde{k}\left(t_{\varepsilon}^{\prime}\right)=\frac{t_{\varepsilon}^{\prime 2}}{2}\left\|v_{\varepsilon}\right\|^{2}-\frac{t_{\varepsilon}^{\prime p}}{p}=\frac{1}{2}\left\|v_{\varepsilon}\right\|^{2+\frac{4}{p-2}}-\frac{1}{p}\left\|v_{\mathcal{\varepsilon}}\right\|^{\frac{2 p}{p-2}}=\frac{p-2}{2 p}\left\|v_{\mathcal{\varepsilon}}\right\|^{\frac{2 p}{p-2}} .
$$


So we already get $\sup _{t \geq 0} \widetilde{k}(t)$. At the same time, we can evaluate $k\left(t_{\varepsilon}\right)$ directly. Combining with (15), we have

$$
\begin{aligned}
k\left(t_{\varepsilon}\right) & =\widetilde{k}\left(t_{\varepsilon}\right)-\int_{\Omega} F\left(x, t_{\varepsilon} v_{\varepsilon}\right) d x \\
& \leq \widetilde{k}\left(t_{\varepsilon}^{\prime}\right)-\int_{\Omega} F\left(x, t_{\varepsilon} v_{\mathcal{E}}\right) d x \\
& =\frac{p-2}{2 p}\left\|v_{\mathcal{\varepsilon}}\right\|^{\frac{2 p}{p-2}}-\int_{\Omega} F\left(x, t_{\varepsilon} v_{\varepsilon}\right) d x \\
& =\frac{p-2}{2 p} A^{\frac{p}{p-2}}+\frac{p-2}{2 p} C_{8} \varepsilon^{\frac{2}{p-2}}-\int_{\Omega} F\left(x, t_{\varepsilon} v_{\mathcal{\varepsilon}}\right) d x
\end{aligned}
$$

Therefore, in order to prove that $\sup _{t \geq 0} I\left(t u^{\prime}\right)<\frac{p-2}{2 p} A^{\frac{p}{p-2}}$, it is sufficient to show that $\frac{p-2}{2 p} C_{8} \varepsilon^{\frac{2}{p-2}}-\int_{\Omega} F\left(x, t_{\varepsilon} v_{\varepsilon}\right) d x<0$ for $\varepsilon$ small enough. To this purpose we prove

$$
\lim _{\varepsilon \rightarrow 0^{+}} \varepsilon^{-\frac{2}{p-2}} \int_{\Omega} F\left(x, t_{\varepsilon} v_{\varepsilon}\right) d x=+\infty .
$$

And we find that Lemma 1 is true if (17) is true. So we can prove (17) instead. In fact, if $f(x, t) \geq m(t)>0$, combining with the definition of $v_{\mathcal{E}}$, (14) and (12), we only need to prove

$$
\lim _{\varepsilon \rightarrow 0^{+}} \varepsilon^{-\frac{2}{p-2}} \int_{|x|<R} M\left(\frac{C \varepsilon^{\frac{1}{p-2}}}{|x| \gamma^{\prime}\left(\varepsilon+|x|^{(p-2) \beta)^{\frac{2}{p-2}}}\right.}\right) d x=+\infty,
$$

where $M(t)=\int_{0}^{t} m(s) d s$ is the primitive function of $m(t)$. Through computation, we have

$$
\begin{aligned}
& \frac{1}{\varepsilon^{\frac{2}{p-2}}} \int_{|x|<R} M\left(\frac{C \varepsilon^{\frac{1}{p-2}}}{|x|^{\gamma^{\prime}}\left(\varepsilon+|x|^{(p-2) \beta)^{\frac{2}{p-2}}}\right.}\right) d x \\
= & \frac{\omega}{\varepsilon^{\frac{2}{p-2}}} \int_{0}^{R} M\left(\frac{C \varepsilon^{\frac{1}{p-2}}}{r \gamma^{\prime}(\varepsilon+r(p-2) \beta)^{\frac{2}{p-2}}}\right) r^{N-1} d r \\
= & \frac{\omega \varepsilon^{\frac{N}{(p-2) \beta}}}{\varepsilon^{\frac{2}{p-2}}} \int_{0}^{R \varepsilon^{-\frac{1}{(p-2) \beta}}} M\left(\frac{C \varepsilon^{-\frac{1}{p-2}-\frac{\gamma^{\prime}}{(p-2) \beta}}}{s^{\gamma^{\prime}}\left(1+s^{(p-2) \beta)^{\frac{2}{p-2}}}\right.}\right) s^{N-1} d s \\
= & \omega \varepsilon^{\frac{N-2 \beta}{(p-2) \beta}} \int_{0}^{R \varepsilon^{-\frac{1}{(p-2) \beta}}} M\left(\frac{C \varepsilon^{-\frac{1}{p-2}-\frac{\gamma^{\prime}}{(p-2) \beta}}}{s^{\gamma^{\prime}}\left(1+s^{(p-2) \beta)^{\frac{2}{p-2}}}\right.}\right) s^{N-1} d s,
\end{aligned}
$$

where $\omega$ is the area of $S^{N-1}$. So we see that (18) is equivalent to

$$
\lim _{\varepsilon \rightarrow 0^{+}} \varepsilon^{\frac{N-2 \beta}{(p-2) \beta}} \int_{0}^{R^{\prime} \varepsilon^{-\frac{1}{(p-2) \beta}}} M\left(\frac{C \varepsilon^{-\frac{1}{p-2}-\frac{\gamma^{\prime}}{(p-2) \beta}}}{s^{\gamma^{\prime}}\left(1+s^{(p-2) \beta)^{\frac{2}{p-2}}}\right.}\right) s^{N-1} d s=+\infty,
$$


for some constant $R^{\prime}>0$. When $R^{\prime} \geq 1$, (19) is equivalent to

$$
\lim _{\varepsilon \rightarrow 0^{+}} \varepsilon^{\frac{N-2 \beta}{(p-2) \beta}} \int_{0}^{\varepsilon^{-\frac{1}{(p-2) \beta}}} M\left(\frac{C \varepsilon^{-\frac{1}{p-2}-\frac{\gamma^{\prime}}{(p-2) \beta}}}{s^{\gamma^{\prime}}\left(1+s^{(p-2) \beta}\right)^{\frac{2}{p-2}}}\right) s^{N-1} d s=+\infty .
$$

Otherwise, when $R^{\prime}<1$, define

$$
M_{\mathcal{E}}=\varepsilon^{\frac{N-2 \beta}{(p-2) \beta}} \int_{R^{\prime} \varepsilon}^{\varepsilon^{-} \frac{1}{(p-2) \beta}} M\left(\frac{C \varepsilon^{-\frac{1}{p-2}-\frac{\gamma^{\prime}}{(p-2) \beta}}}{s^{\prime}\left(1+s^{(p-2) \beta)^{\frac{2}{p-2}}}\right.}\right) s^{N-1} d s,
$$

for $m(t)>0$ and note that for some constant $C_{9}$, we have

$$
\left|M_{\varepsilon}\right| \leq C_{9} \varepsilon^{\frac{N-2 \beta}{(p-2) \beta}} M\left(C_{10} \varepsilon^{\frac{1}{p-2}}\right) \varepsilon^{-\frac{N}{(p-2) \beta}}
$$

By $\left(f_{5}\right)$ and $f(x, t) \geq m(t)$, as $\varepsilon \rightarrow 0$ we know

$$
M\left(C_{10} \varepsilon^{\frac{1}{p-2}}\right) \leq \frac{1}{2} \lambda\left(C_{10} \varepsilon^{\frac{1}{p-2}}\right)^{2} \leq C_{11} \varepsilon^{\frac{2}{p-2}},
$$

and then we can get

$$
\left|M_{\mathcal{\varepsilon}}\right| \leq C_{12} \varepsilon^{\frac{N-2 \beta}{(p-2) \beta}} \varepsilon^{\frac{2}{p-2}-\frac{N}{(p-2) \beta}} \leq C_{12}
$$

which means that $M_{\varepsilon}$ is bounded as $\varepsilon$ is small, and then (19) is also equivalent to (20). So we only need prove that (20) is true.

Last, we prove that (20) is true if one of $(a),(b)$ and (c) hold.

In case $(a)$ : From $(a)$, we know

$f(x, t) \geq \sigma \chi_{I}(t) \triangleq m(t)$ for almost everywhere $x \in \omega$ and for all $t \geq 0$ where $\chi_{I}$ is the characteristic function of $I(I \subset(0,+\infty))$. Thus we get

$$
M(t) \geq \eta>0 \text { for all } t \geq B,
$$

for some constants $\eta>0$ and $B>0$. Then we have

$$
M\left(\frac{C \varepsilon^{-\frac{1}{p-2}-\frac{\gamma^{\prime}}{(p-2) \beta}}}{s \gamma^{\prime}\left(1+s^{(p-2) \beta)^{\frac{2}{p-2}}}\right.}\right) \geq \eta \text { for all } s \text { such that } s \leq C_{13} \varepsilon^{-\frac{\beta+\gamma^{\prime}}{(p-2)\left(2 \beta+\gamma^{\prime}\right) \beta}}
$$

where $C_{13}$ (relate to $B$ ) is a constant and $\varepsilon$ is small. Then we have

$$
\begin{aligned}
& \varepsilon^{\frac{N-2 \beta}{(p-2) \beta}} \int_{0}^{\varepsilon^{-\frac{1}{(p-2) \beta}}} M\left(\frac{C_{\varepsilon^{-\frac{1}{p-2}}-\frac{\gamma^{\prime}}{(p-2) \beta}}}{s^{\gamma^{\prime}}\left(1+s^{(p-2) \beta)^{\frac{2}{p-2}}}\right.}\right) s^{N-1} d s \\
& \geq \varepsilon^{\frac{N-2 \beta}{(p-2) \beta}} \int_{1}^{\varepsilon^{-\frac{1}{(p-2) \beta}}} M\left(\frac{C^{-\frac{1}{p-2}-\frac{\gamma^{\prime}}{(p-2) \beta}}}{s^{\gamma^{\prime}}\left(1+s^{(p-2) \beta}\right)^{\frac{2}{p-2}}}\right) s^{N-1} d s \\
& \geq \eta \varepsilon^{\frac{N-2 \beta}{(p-2) \beta}} \int_{1}^{C_{13} \varepsilon^{-\frac{\beta+\gamma^{\prime}}{(p-2)\left(2 \beta+\gamma^{\prime}\right) \beta}}} s^{N-1} d s \\
& =C_{14} \varepsilon^{\frac{N-2 \beta}{(p-2) \beta}-\frac{\left(\beta+\gamma^{\prime}\right) N}{(p-2)\left(2 \beta+\gamma^{\prime}\right) \beta}} \\
& =C_{14} \varepsilon^{\frac{N-\left(4 \beta+2 \gamma^{\prime}\right)}{(p-2)\left(2 \beta+\gamma^{\prime}\right)}}
\end{aligned}
$$


when $N<\left(4 \beta+2 \gamma^{\prime}\right)$ (It is same as $\left.N>2 a+2+2 \sqrt{\mu+(1+a)^{2}}\right)$. It is said that (20) is true.

In case $(b)$ : From $(b)$, we know

$$
f(x, t) \geq \sigma t \chi_{I}(t) \triangleq m(t) \text { for almost everywhere } x \in \omega \text { and for all } t \geq 0
$$

where $I$ is either $[0, D]$ or $[D,+\infty](D \geq 1)$. Thus we can get either

$$
M(t)=\frac{1}{2} \eta t^{2} \text { for } 0 \leq t \leq D
$$

or

$$
M(t)=\frac{1}{2} \eta\left(t^{2}-D^{2}\right) \text { for } t \geq D
$$

When (21) holds, we have

$$
\begin{aligned}
& \varepsilon^{\frac{N-2 \beta}{(p-2) \beta}} \int_{0}^{\varepsilon^{-\frac{1}{(p-2) \beta}}} M\left(\frac{C \varepsilon^{-\frac{1}{p-2}-\frac{\gamma^{\prime}}{(p-2) \beta}}}{s \gamma^{\prime}\left(1+s^{(p-2) \beta)^{\frac{2}{p-2}}}\right.}\right) s^{N-1} d s
\end{aligned}
$$

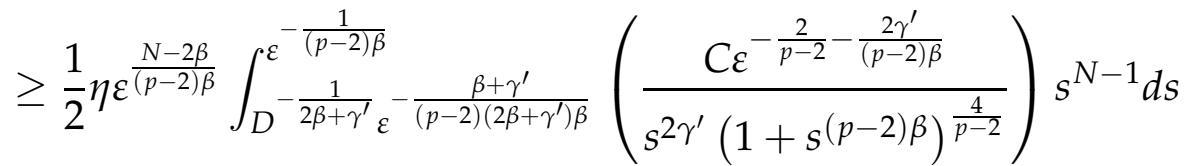

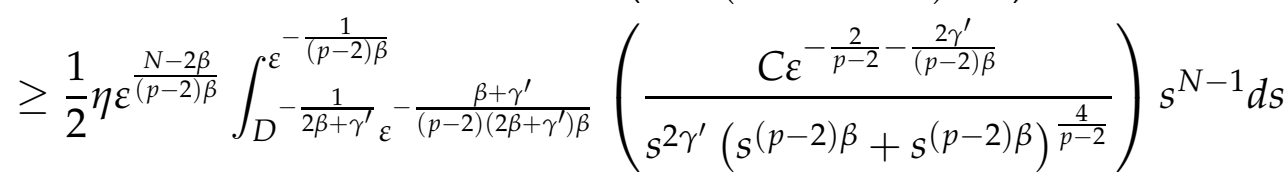

$$
\begin{aligned}
& =\frac{C}{2 N} \eta\left(\frac{1}{2}\right)^{\frac{4}{p-2}}\left(\ln \left(\varepsilon^{-\frac{N}{(p-2) \beta}}\right)-\ln \left(\varepsilon^{-\frac{\left(\beta+\gamma^{\prime}\right) N}{(p-2)\left(2 \beta+\gamma^{\prime}\right) \beta}}\right)\right)-\frac{1}{2} \eta \ln \left(D^{-\frac{1}{2 \beta+\gamma^{\prime}}}\right) \\
& =\frac{C}{2} \eta\left(\frac{1}{2}\right)^{\frac{4}{p-2}}\left(-\frac{1}{(p-2) \beta}+\frac{\beta+\gamma^{\prime}}{(p-2)\left(2 \beta+\gamma^{\prime}\right) \beta}\right) \ln \varepsilon+\frac{\eta}{4 \beta+2 \gamma^{\prime}} \ln D \\
& \geq 2^{-\frac{p+2}{p-2}} C \eta \frac{1}{(p-2)\left(2 \beta+\gamma^{\prime}\right)}|\ln \varepsilon| \text {, }
\end{aligned}
$$

as $\varepsilon \rightarrow 0^{+}$and $N=4 \beta+2 \gamma^{\prime}$ (It is same as $N=2 a+2+2 \sqrt{\mu+(1+a)^{2}}$ ). 
When (22) holds, we have (for some constant $C_{15}$ relate to $D$ )

$$
\begin{aligned}
& \varepsilon^{\frac{N-2 \beta}{(p-2) \beta}} \int_{0}^{\varepsilon^{-\frac{1}{(p-2) \beta}}} M\left(\frac{C \varepsilon^{-\frac{1}{p-2}-\frac{\gamma^{\prime}}{(p-2) \beta}}}{s \gamma^{\prime}\left(1+s^{(p-2) \beta)^{\frac{2}{p-2}}}\right.}\right) s^{N-1} d s \\
& \geq \varepsilon^{\frac{N-2 \beta}{(p-2) \beta}} \int_{1}^{\varepsilon^{-\frac{1}{(p-2) \beta}}} M\left(\frac{C \varepsilon^{-\frac{1}{p-2}-\frac{\gamma^{\prime}}{(p-2) \beta}}}{s \gamma^{\prime}\left(1+s^{(p-2) \beta)^{\frac{2}{p-2}}}\right.}\right) s^{N-1} d s \\
& \geq \frac{1}{4} \eta \mathcal{E}^{\frac{N-2 \beta}{(p-2) \beta}} \int_{1}^{C_{15} \varepsilon^{-\frac{\beta+\gamma^{\prime}}{(p-2)\left(2 \beta+\gamma^{\prime}\right) \beta}}}\left(\frac{C \varepsilon^{-\frac{2}{p-2}-\frac{2 \gamma^{\prime}}{(p-2) \beta}}}{s^{2 \gamma^{\prime}}\left(1+s^{(p-2) \beta)^{\frac{4}{p-2}}}\right.}\right) s^{N-1} d s \\
& \geq \frac{1}{4} \eta \varepsilon^{\frac{N-2 \beta}{(p-2) \beta}} \int_{1}^{C_{15} \varepsilon^{-\frac{\beta+\gamma^{\prime}}{(p-2)\left(2 \beta+\gamma^{\prime}\right) \beta}}}\left(\frac{C \varepsilon^{-\frac{2}{p-2}-\frac{2 \gamma^{\prime}}{(p-2) \beta}}}{s^{2 \gamma^{\prime}}\left(s^{(p-2) \beta}+s^{(p-2) \beta)^{\frac{4}{p-2}}}\right.}\right) s^{N-1} d s \\
& \geq \frac{1}{4 N} \eta 2^{-\frac{4}{p-2}} \ln \left(C_{15} \varepsilon^{-\frac{\left(\beta+\gamma^{\prime}\right) N}{(p-2)\left(2 \beta+\gamma^{\prime}\right) \beta}}\right) \\
& \geq 2^{-\frac{2 p}{p-2}} \frac{\left(\beta+\gamma^{\prime}\right) \eta}{(p-2)\left(2 \beta+\gamma^{\prime}\right) \beta}|\ln \varepsilon|+2^{-\frac{2 p}{p-2}} \ln \left(C_{15}\right),
\end{aligned}
$$

as $\varepsilon \rightarrow 0^{+}$and $N=4 \beta+2 \gamma^{\prime}$ (It is same as $N=2 a+2+2 \sqrt{\mu+(1+a)^{2}}$ ). So either (21) or (22) holds, (20) is true.

In case $(c)$ : Setting $m(t)=\inf _{x \in \omega} f(x, t)$, so that $\lim _{t \rightarrow+\infty} \frac{m(t)}{t^{\frac{N-\gamma^{\prime}-3 \beta}{\gamma^{\prime}+\beta}}}=+\infty$ and $f(x, t) \geq$ $m(t)$ for almost everywhere $x \in \omega$ and for all $t \geq 0$. Therefore from (c), we can get for all $\theta>0$, there is a constant $D>0$ such that $M(t) \geq \theta t^{\frac{N-2 \beta}{\gamma^{\prime}+\beta}}$ for all $t \geq D$. And then we have (for some constant $C_{16}>0$ relate to $D$ and $\varepsilon$ small)

$$
\begin{aligned}
& \varepsilon^{\frac{N-2 \beta}{(p-2) \beta}} \int_{0}^{\varepsilon^{-\frac{1}{(p-2) \beta}}} M\left(\frac{C \varepsilon^{-\frac{1}{p-2}-\frac{\gamma^{\prime}}{(p-2) \beta}}}{s \gamma^{\prime}\left(1+s^{(p-2) \beta)^{\frac{2}{p-2}}}\right.}\right) s^{N-1} d s \\
& \geq \varepsilon^{\frac{N-2 \beta}{(p-2) \beta}} \int_{1}^{\varepsilon^{-\frac{1}{(p-2) \beta}}} M\left(\frac{C \varepsilon^{-\frac{1}{p-2}-\frac{\gamma^{\prime}}{(p-2) \beta}}}{s \gamma^{\prime}(1+s(p-2) \beta)^{\frac{2}{p-2}}}\right) s^{N-1} d s
\end{aligned}
$$

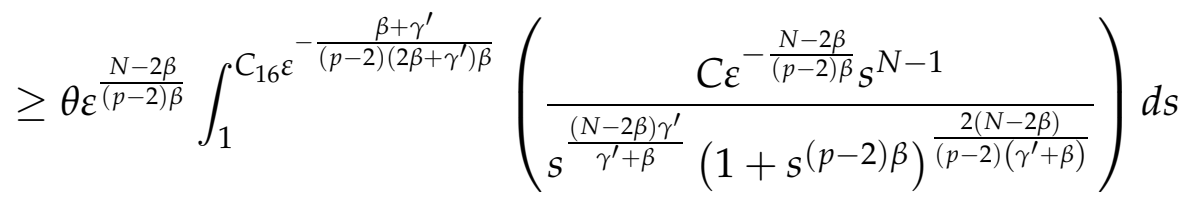

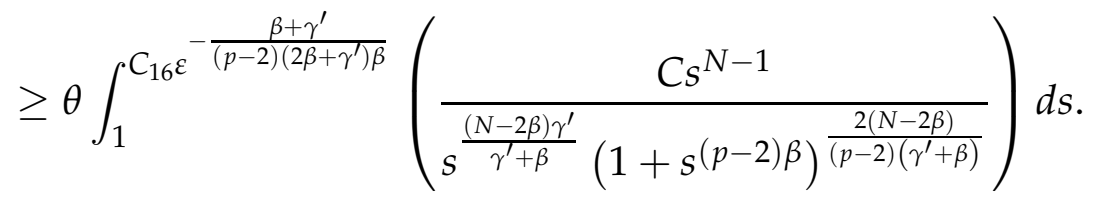


When $N>4 \beta+2 \gamma^{\prime}$, we can compute

$$
\begin{aligned}
& \frac{(N-2 \beta) \gamma^{\prime}}{\gamma^{\prime}+\beta}+\frac{2(N-2 \beta) \beta}{\gamma^{\prime}+\beta}-(N-1) \\
& =\frac{(N-2 \beta)\left(\gamma^{\prime}+2 \beta\right)-(N-1)\left(\gamma^{\prime}+\beta\right)}{\gamma^{\prime}+\beta} \\
& =\frac{N \beta-2 \beta \gamma^{\prime}-4 \beta^{2}+\beta+\gamma^{\prime}}{\gamma^{\prime}+\beta} \\
& >\frac{\left(4 \beta+2 \gamma^{\prime}\right) \beta-2 \beta \gamma^{\prime}-4 \beta^{2}+\beta+\gamma^{\prime}}{\gamma^{\prime}+\beta} \\
& =1 .
\end{aligned}
$$

Then, there exists a constant $G$, so that we have

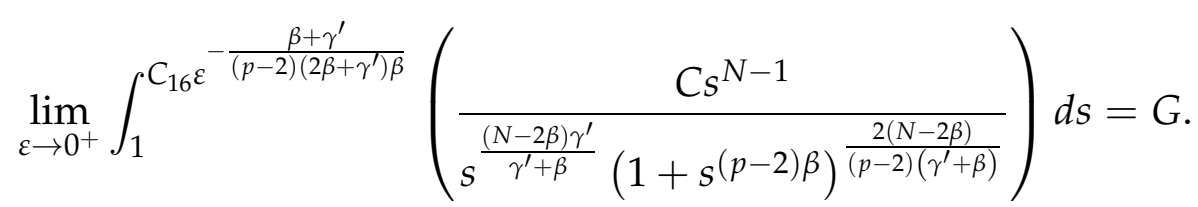

Hence we obtain

$$
\lim _{\varepsilon \rightarrow 0^{+}} \varepsilon^{\frac{N-2 \beta}{(p-2) \beta}} \int_{0}^{\varepsilon^{-\frac{1}{(p-2) \beta}}} M\left(\frac{C \varepsilon^{-\frac{1}{p-2}-\frac{\gamma^{\prime}}{(p-2) \beta}}}{s \gamma^{\prime}\left(1+s^{(p-2) \beta)^{\frac{2}{p-2}}}\right.}\right) s^{N-1} d s>G \theta .
$$

Consequently, from the arbitrariness of $\theta>0,(20)$ is true. Thus (20) holds for one of $(a),(b)$ and $(c)$. This completes the proof of Lemma 1.

As for all $\theta>0$ and $N>4 \beta+2 \gamma^{\prime}$ (It is same as $N<2 a+2+2 \sqrt{\mu+(1+a)^{2}}$ ), this means that (20) is true. From these above, we completes the proof of Lemma 1.

Lemma 2. Assume $0 \leq a<\sqrt{\bar{\mu}}, a \leq b<a+1,0<\lambda<\lambda_{1}$, ( $\left.f_{5}\right)$ hold, then problem (8) has a nontrivial and nonnegative solution.

Proof. We claim that $I$ satisfies the mountain-pass geometrical structure on $H$. Indeed, for all $t>0$, one has

$$
I(t u)=\frac{t^{2}}{2}\|u\|^{2}-\frac{t^{p}}{p} \int_{\Omega} \frac{\left(u^{+}\right)^{p}}{|x|^{b p}} d x-\int_{\Omega} F\left(x, t u^{+}\right) d x .
$$

Consequently, we have $\lim _{t \rightarrow 0^{+}} I(t u)=0$ and $\lim _{t \rightarrow+\infty} I(t u)=-\infty$. Moreover, from $\left(f_{5}\right)$ and $p>2$, there exists a suitable $t_{0}>0$ such that $I\left(t_{0} u\right)>0$. Then our claim is true. Therefore, according to Lemma 1, we obtain that $I$ has a (PS) sequence on $H$ for all $0<c<\frac{p-2}{2 p} A^{\frac{p}{p-2}}$. Without loss of generality, we may assume that $\left\{u_{n}\right\}$ is a (PS) $)_{c}$ sequence of $I$. Now, we prove that $\left\{u_{n}\right\}$ is bounded in $H$. It follows from $\left(f_{5}\right)$ and the boundedness of $\Omega$, for any $\varepsilon, \varepsilon_{4}>0$, there exists $M>0$, such that

$$
\begin{aligned}
& |F(x, t)| \leq \varepsilon_{4}|t|^{p}, \quad x \in \Omega, \quad|t|>M ; \quad|F(x, t)| \leq C_{17}(\varepsilon), \quad|t| \in[0, M] ; \\
& |f(x, t) t| \leq \varepsilon_{4} p|t|^{p}, \quad x \in \Omega, \quad|t|>M ; \quad|f(x, t) t| \leq C_{18}(\varepsilon), \quad|t| \in[0, M] \text {. }
\end{aligned}
$$


Therefore, we have

$$
\begin{array}{r}
|F(x, t)| \leq C_{17}(\varepsilon)+\varepsilon_{4}|t|^{p}, \quad|f(x, t) t| \leq C_{18}(\varepsilon)+p \varepsilon_{4}|t|^{p}, \\
\text { for any }(x, t) \in \Omega \times \mathbb{R} .
\end{array}
$$

Let $C_{11}(\varepsilon)=C_{17}(\varepsilon)+\frac{1}{\xi} C_{18}(\varepsilon)$ and $\varepsilon=\varepsilon_{4}+\frac{p}{\xi} \varepsilon_{4}$, where $\xi \in(2, p)$. Combining with (23) we get

$$
\begin{aligned}
F(x, t)-\frac{1}{2} f(x, t) t \leq F(x, t)-\frac{1}{\xi} f(x, t) t \leq C_{19}(\varepsilon)+\varepsilon|t|^{p} & \\
& \text { for any }(x, t) \in \Omega \times \mathbb{R} .
\end{aligned}
$$

We observe that $h(x, t) \triangleq|x|^{-b p}|t|^{p-1}+f(x, t)$ satisfies the Ambrosetti-Rabinowitz condition. By (24), we can get

$$
\begin{aligned}
\xi H(x, t)-h(x, t) t & =\left(\frac{\xi}{p}-1\right)|x|^{-b p}|t|^{p}+(\xi F(x, t)-f(x, t) t) \\
& \leq\left(|x|^{-b p}\left(\frac{\xi}{p}-1\right)+\xi \varepsilon\right)|t|^{p}+\xi C_{11}(\varepsilon)
\end{aligned}
$$

so for $\varepsilon$ sufficiently small, there exists $M_{2}>0$, such that

$$
0 \leq \xi H(x, t) \leq h(x, t) t, \quad t \geq M_{2}
$$

where $H(x, t)=\int_{0}^{t} h(x, s) d s$. Moreover, by $\left(f_{5}\right)$, we get

$$
H(x, t)-\frac{1}{\xi} h(x, t) t \leq \max _{x \in \Omega, 0 \leq t \leq M_{2}}\left(F(x, t)-\frac{1}{\xi} f(x, t) t\right) \triangleq M_{3} .
$$

It follows from two inequalities above that

$$
H(x, t)-\frac{1}{\xi} h(x, t) t \leq M_{3} \text {, for all } x \in \bar{\Omega} \backslash\{0\}, \quad t \geq 0 .
$$

Since $\left\{u_{n}\right\}$ is a (PS) $c$ sequence in $H$, by (25) we have

$$
\begin{aligned}
c+1+o(1)\left\|u_{n}\right\| & \geq I\left(u_{n}\right)-\frac{1}{\xi}\left\langle I^{\prime}\left(u_{n}\right), u_{n}\right\rangle \\
& =\left(\frac{1}{2}-\frac{1}{\xi}\right)\left\|u_{n}\right\|^{2}+\left(\frac{1}{\xi}-\frac{1}{p}\right) \int_{\Omega} \frac{\left(u_{n}^{+}\right)^{p}}{|x|^{b p}} d x- \\
& =\left(\frac{1}{2}-\frac{1}{\xi}\right)\left\|u_{n}\right\|^{2}-\int_{\Omega}\left(H\left(x, u_{n}^{+}\right)-\frac{1}{\xi} f\left(x, u_{n}^{+}\right)-\frac{1}{\xi} h\left(x, u_{n}^{+}\right) u_{n}\right) d x \\
& \geq\left(\frac{1}{2}-\frac{1}{\xi}\right)\left\|u_{n}\right\|^{2}-M_{3} \mid \Omega
\end{aligned}
$$


Hence we conclude that $\left\{u_{n}\right\}$ is a bounded sequence in $H$. By the continuity of embedding, we have $\left\|u_{n}\right\|_{L^{2^{*}}(\Omega)}^{2^{*}} \leq C_{20}<+\infty$. Going if necessary to a subsequence, one can get

$$
\left\{\begin{array}{l}
u_{n} \rightarrow u, \text { weakly in } H, \quad \text { as } n \rightarrow \infty, \\
u_{n} \rightarrow u, \text { in } L^{r}(\Omega), 1<r<2^{*}, \text { as } n \rightarrow \infty, \\
u_{n} \rightarrow u, \text { a.e. in } \Omega, \quad \text { as } n \rightarrow \infty
\end{array}\right.
$$

Next, we must prove that $u \not \equiv 0$, and $u$ is a weak solution of problem (8).

First, we prove that $u \neq \equiv 0$. Indeed, suppose that $u \equiv 0$ and we can also get $I(u)=0$. It follows from $\left(f_{5}\right)$ and the boundedness of $\Omega$, for any $\varepsilon>0$, there exist $M_{4}$ and $C_{21}(\varepsilon)>0$ such that

$$
|f(x, t) t| \leq \frac{1}{2 C_{20}} \varepsilon|t|^{p}, \quad x \in \Omega,|t|>M_{4} ; \quad|f(x, t) t| \leq C_{21}(\varepsilon), \quad x \in \Omega,|t| \in\left[0, M_{4}\right] .
$$

Therefore we have

$$
|f(x, t) t| \leq \frac{1}{2 C_{20}} \varepsilon|t|^{p}+C_{21}(\varepsilon), \quad(x, t) \in \bar{\Omega} \times \mathbb{R}^{+} .
$$

By the Vitali Convergence Theorem in [8], combining with (26) and use the same method like [6], we have

$$
\int_{\Omega} f\left(x, u_{n}^{+}\right) u_{n} d x \rightarrow \int_{\Omega} f\left(x, u^{+}\right) u d x, \quad n \rightarrow \infty .
$$

and

$$
\int_{\Omega} F\left(x, u_{n}^{+}\right) d x \rightarrow \int_{\Omega} F\left(x, u^{+}\right) d x, \quad n \rightarrow \infty
$$

Since $I^{\prime}\left(u_{n}\right) \rightarrow 0$ in $H^{*}$, we obtain

$$
\left\langle I^{\prime}\left(u_{n}^{+}\right), u_{n}\right\rangle=\left\|u_{n}\right\|^{2}-\int_{\Omega} \frac{\left(u_{n}^{+}\right)^{p}}{|x|^{b p}} d x-\int_{\Omega} f\left(x, u_{n}^{+}\right) u_{n} d x=o(1) .
$$

Since $I\left(u_{n}\right) \rightarrow c(n \rightarrow \infty)$, together with (28) and $u \equiv 0$, we obtain

$$
\begin{aligned}
I\left(u_{n}\right) & =\frac{1}{2}\left\|u_{n}\right\|^{2}-\frac{1}{p} \int_{\Omega} \frac{\left(u_{n}^{+}\right)^{p}}{|x|^{b p}} d x-\int_{\Omega} F\left(x, u_{n}{ }^{+}\right) d x \\
& =\frac{1}{2}\left\|u_{n}\right\|^{2}-\frac{1}{p} \int_{\Omega} \frac{\left(u_{n}^{+}\right)^{p}}{|x|^{b p}} d x+o(1) \\
& =c+o(1) .
\end{aligned}
$$

Therefore, we get

$$
\frac{1}{2}\left\|u_{n}\right\|^{2}-\frac{1}{p} \int_{\Omega} \frac{\left(u_{n}^{+}\right)^{p}}{|x|^{b p}} d x=c+o(1)
$$


For $u \equiv 0$, from (27) and (29), we have

$$
\left\|u_{n}\right\|^{2}-\int_{\Omega} \frac{\left(u_{n}^{+}\right)^{p}}{|x|^{b p}} d x=o(1)
$$

Extracting still another sequence (still denoted by $\left\{u_{n}\right\}$ ) we may assume

$$
\lim _{n \rightarrow \infty}\left\|u_{n}\right\|^{2}=s, \quad \lim _{n \rightarrow \infty} \int_{\Omega} \frac{\left(u_{n}^{+}\right)^{p}}{|x|^{b p}} d x=s,
$$

where $s \geq 0$ is a constant. At the same time, as we all known the best HardySobolev constant, we deduce that

$$
\left\|u_{n}\right\|^{2} \geq A\left(\int_{\Omega} \frac{\left(u_{n}^{+}\right)^{p}}{|x|^{b p}} d x\right)^{\frac{2}{p}}, \quad \text { for all } n \in Z_{+},
$$

then $s \geq A s^{\frac{2}{p}}$, so $s \geq A^{\frac{p}{p-2}}$. Consequently, together with (30) and (31), one has

$$
0=c-\frac{1}{2} s+\frac{1}{p} s=c-\left(\frac{1}{2}-\frac{1}{p}\right) s \leq c-\frac{p-2}{2 p} A^{\frac{p}{p-2}}<0,
$$

which is a contradiction. Therefore, we get $u \not \equiv 0$.

Second, we prove that $u$ is a weak solution of problem (8). For any $v \in H$, combining with (27) and $I^{\prime}\left(u_{n}\right) \rightarrow 0$, we have

$$
\begin{aligned}
0 & =\lim _{n \rightarrow \infty}\left\langle I^{\prime}\left(u_{n}\right), v\right\rangle \\
& =\int_{\Omega}\left(|x|^{-2 a}(\nabla u, \nabla v)-\mu \frac{u v}{|x|^{2(1+a)}}\right) d x-\int_{\Omega} \frac{\left(u^{+}\right)^{p-1} v}{|x|^{b p}} d x-\int_{\Omega} f\left(x, u^{+}\right) v d x \\
& =\left\langle I^{\prime}(u), v\right\rangle .
\end{aligned}
$$

It means that $u$ is a weak solution of problem (8). This completes the proof of Lemma 2.

Proof of Theorem 4. From the Hardy-Sobolev inequalities and Caffarelli-KohnNirenberg inequalities, we can easily get

$$
\|u\|_{L^{2}}^{2} \leq \frac{1}{\lambda_{1}}\|u\|^{2}, \quad \int_{\Omega} \frac{(u)^{p}}{|x|^{b p}} d x \leq C_{22}\|u\|^{p}, \quad\|u\|_{L^{p}}^{p} \leq C_{23}\|u\|^{p},
$$

for all $u \in H$. It follows from $\left(f_{5}\right)$ that there exist $\delta_{1}, \delta_{2}>0$ and $\delta_{2}<\delta_{1}$ such that

$$
|F(x, t)|<t^{p}, \text { for } t>\delta_{1}
$$

and

$$
|F(x, t)|<\frac{1}{2} \lambda t^{2}, \text { for } 0<t<\delta_{2} .
$$

As $f \in C\left(\Omega \times \mathbb{R}^{+}, \mathbb{R}\right)$, there exists $M_{5}>0$, such that

$$
|F(x, t)| \leq M_{5}, \text { for all } t \in\left[\delta_{2}, \delta_{1}\right],
$$


for all $x \in \bar{\Omega}$. Therefore, we have

$$
|F(x, t)| \leq \frac{1}{2} \lambda t^{2}+t^{p}+M_{5} \leq \frac{1}{2} \lambda t^{2}+\left(1+M_{5} \delta_{2}^{-p}\right) t^{p},
$$

for all $t \in \mathbb{R}^{+}$and for $x \in \bar{\Omega}$. Then one gets

$$
|F(x, t)| \leq \frac{1}{2} \lambda|t|^{2}+C_{24}|t|^{p}
$$

for all $t \in \mathbb{R}$ and for $x \in \bar{\Omega}$, where $C_{16}=1+M_{5} \delta_{2}^{-p}$. By (32) and (33), we have

$$
\begin{aligned}
I(u) & =\frac{1}{2}\|u\|^{2}-\frac{1}{p} \int_{\Omega} \frac{\left(u^{+}\right)^{p}}{|x|^{b p}} d x-\int_{\Omega} F\left(x, u^{+}\right) d x \\
& \geq \frac{1}{2}\|u\|^{2}-\frac{C_{22}}{p}\|u\|^{p}-\frac{\lambda}{2}\|u\|_{L^{2}}^{2}-C_{24}\|u\|_{L^{p}}^{p} \\
& \geq \frac{1}{2}\|u\|^{2}-\frac{C_{22}}{p}\|u\|^{p}-\frac{\lambda}{2 \lambda_{1}}\|u\|^{2}-C_{23} C_{24}\|u\|^{p}
\end{aligned}
$$

for $0<\lambda<\lambda_{1}$, there exists $\alpha>0$ such that $I(u) \geq \alpha$ for all $u \in \partial B_{r}=$ $\{u \in H,\|u\|=r\}$, where $r>0$ sufficiently small. By Lemma 1 there exists $u^{\prime} \in H, u^{\prime} \not \equiv 0$, such that

$$
\sup _{t \geq 0} I\left(t u^{\prime}\right)<\frac{p-2}{2 p} A^{\frac{p}{p-2}} .
$$

It follows from $f_{5}$ that $\lim _{t \rightarrow+\infty} I\left(t u^{\prime}\right) \rightarrow-\infty$. Hence we can choose $t^{\prime}>0$ such that $\left\|t^{\prime} u^{\prime}\right\|>r$ and $I\left(t^{\prime} u^{\prime}\right) \leq 0$. Applying the Mountain Pass Lemma in [7], there is a sequence $\left\{u_{n}\right\} \subset H$ satisfying

$$
I\left(u_{n}\right) \rightarrow c \geq \alpha \quad \text { and } \quad I^{\prime}\left(u_{n}\right) \rightarrow 0,
$$

where

$$
\begin{aligned}
& c=\inf _{h \in \tau} \max _{t \in[0,1]} I(h(t)) \\
& \tau=\left\{h \in([0,1], H) \mid h(0)=0, h(1)=t^{\prime} u^{\prime}\right\} .
\end{aligned}
$$

Note that

$$
0<\alpha \leq c=\inf _{h \in \tau} \max _{t \in[0,1]} I(h(t)) \leq \max _{t \in[0,1]} I\left(t t^{\prime} u^{\prime}\right) \leq \sup _{t \geq 0} I\left(t u^{\prime}\right)<\frac{p-2}{2 p} A^{\frac{p}{p-2}} .
$$

Now Lemma 2 suggests $\left\{u_{n}\right\} \subset H$ has a convergent subsequence (still denoted by $\left.\left\{u_{n}\right\}\right)$. Assume that $\left\{u_{n}\right\}$ converges to $u \in H$. From the continuity of $I^{\prime}$, we know that $u$ is a weak solution of problem (8). It yields that $u \geq 0$ from $\left\langle I^{\prime}(u), u^{-}\right\rangle=0$, where $u^{-}=\min \{u, 0\}$. By the strong maximum principle, we get that $u$ is a positive solution of problem (8). So the proof of Theorem 4 is completed. 


\section{References}

[1] L. Caffarelli, R. Kohn, L. Nirenberg, First order interpolation inequality with weights, Compos. Math. 53 (1984) 259-275.

[2] K. Chou, C. Chu, On the best constant for a weighted Sobolev-Hardy inequality, J. Lond. Math. Soc. (2) 48 (1993) 137-151.

[3] F. Catrina, Z. Wang, On the Caffarelli-Kohn-Nirenberg inequalities: Sharp constants, existence (and nonexistence), and symmetry of external functions, Comm. Pure Appl. Math. 54 (2) (2001) 229-258.

[4] X.J. Huang, X.P. Wu, C.L. Tang, Multiple positive solutions for semilinear elliptic equations with critical weighted Hardy-Sobolev exponents, Nonlinear Anal. 74 (2011) 2602-2611

[5] L. Ding, C.L. Tang, Existence and multiplicity of positive solutions for a class of semilinear elliptic equations involving Hardy term and Hardy-Sobolev critical exponents, J. Math. Anal. Appl. 339 (2008) 1073-1083.

[6] L. Huang, X.P. Wu, C.L. Tang, Existence and multiplicity of solutions for semilinear elliptic equations with critical weighted Hardy-Sobolev exponents, Nonlinear Anal. 71 (2009) 1916-1924.

[7] P. H. Rabinowitz. Minimax methods in critical point theory with applications to differential equations, Conference Board of the Mathematical Sciences, vol. 65 Providence, RI: American Mathematical Society, 1986.

[8] W. Rudin, Real and Complex Analysis, 3rd ed., McGraw-Hill, New York, 1987.

[9] H. Brezis, L. Nirenberg, Positive solutions of nonlinear elliptic equations involving critical Sobolev exponents, Comm. Pure Appl. Math. 36 (4) (1983) 437-477.

[10] D. Kang, G. Li, S. Peng, Positive solutions and critical dimensions for the elliptic problems involving the Caffarelli-Kohn-Nirenberg inequalities, J. Jilin. Univ. Sci. 46 (2008) 423-427.

[11] N. Ghoussoub, C. Yuan, Multiple solutions for quasilinear PDEs involving the critical Sobolev and Hardy exponents, Trans. Amer. Math. Soc. 352 (2000) 5703-5743.

[12] B. Abdellaoui, I. Peral, Some results for semilinear elliptic equations with critical potential, Proc. Roy. Soc. Edinburgh Sect. A 132 (1) (2002) 1-24.

[13] D. Cao, P. Han, Solutions for semilinear elliptic equations with critical exponents and Hardy potential, J. Differential Equations 205 (2) (2004) 521-537.

[14] A. Ferrero, F. Gazzola, Existence of solutions for singular critical growth semi-linear elliptic equations, J. Differential Equations 177 (2) (2001) 494-522. 
[15] J.P. Garcia Azorero, I. Peral Alonso, Hardy inequalities and some critical elliptic and parabolic problems, J. Differential Equations 144 (2) (1998) 441-476.

[16] S. Terracini, On positive entire solutions to a class of equations with a singular coefficient and critical exponent, Adv. Differential Equations 1 (2) (1996) 241-264.

[17] L. Ding, C.L. Tang, Existence and multiplicity of solutions for semilinear elliptic equations with Hardy terms and Hardy-Sobolev critical exponents, Appl. Math. Lett. 20 (12) (2007) 1175-1183.

[18] N. Ghoussoub, X.S. Kang, Hardy-Sobolev critical elliptic equations with boundary singularities, Ann. Inst. H. Poincar Anal. Non Linaire 21 (6) (2004) 767-793.

[19] D. Kang, S. Peng, Positive solutions for singular critical elliptic problems, Appl. Math. Lett. 17 (4) (2004) 411-416.

[20] D. Kang, S. Peng, Solutions for semilinear elliptic problems with critical Sobolev-Hardy exponents and Hardy potential, Appl. Math. Lett. 18 (10) (2005) 1094-1100.

[21] Z.F. Shen, M.B. Yang, Nontrivial solutions for Hardy-Sobolev critical elliptic equations, Acta. Math. Sinica (Chin. Ser.) 48 (5) (2005) 999-1010.

[22] D. Kang, On the elliptic problems with critical weighted Sobolev-Hardy exponents, Nonlinear Anal. 66 (5) (2007) 1037-1050.

[23] M. Lin, Some further results for a class of weighted nonlinear elliptic equations, J. Math. Anal. Appl. 337 (1) (2008) 537-546.

Department of Mathematics and Physics,

Officers College Of PAPF,

Chengdu 610213, People's Republic of China

and

School of Mathematics and Statistics,

Southwest University,

Chongqing 400715, People's Republic of China

Tel.: +86 23 68253135; fax: +86 2368253135 .

E-mail address: tangcl@swu.edu.cn 